\section{Integrative Medicine \\ International}

\title{
The Brief Theory of Viscus and Its Manifestations in Traditional Chinese Medicine
}

\author{
Weilong Liao Cuijiao Dang Weidong Pan \\ Department of Neurology, Shuguang Hospital Affiliated to Shanghai University of \\ Traditional Chinese Medicine, Shanghai, China
}

\section{Keywords}

Zang organs · Fu organs · Traditional Chinese medicine · Zangxiang · Constituents and orifices · Five elements theory

\section{Introduction}

Traditional Chinese medicine (TCM) includes the quintessence of classical Chinese philosophy, culture, and science from ancient Chinese history [1]. The earliest description of viscus in TCM is found in the handbook Huangdi Neijing, which termed viscus as zangxiang (藏象). “zang” (臟) refers to internal organ in Chinese, “xiang” (象) means manifestation of image or phenomenon. When used together, zangxiang refers to internal organs and the external manifestations of their physiological and pathological visualizations and states. The theory of zangxiang mainly studies the zang organs and the fu organs. That is why it is also known as "the theory of zang-fu organs" (藏腑學說) [2]. According to the ancient theory, all internal organs are divided into 2 major categories, namely the 5 zang organs and the $6 \mathrm{fu}$ organs. The 5 zang organs include the heart (心), the liver (肝), the spleen (脾), the lung (肺), and the kidney (腎), and the 6 fu organs include the gallbladder (膽), the stomach (胃), the small intestine (小腸), the large intestine (大腸), the urinary bladder (膀胱), and sanjiao (the triple energizer). Besides, there is another group of tissues and organs, similar to the zang organs in function and the fu organs in form, which is termed the extraordinary fu organs, including the brain (腦), the marrow (髓), the bones (骨), the vessels (脈), the gallbladder (膽), and the uterus (女子胞) [3].

The content of the zangxiang theory is composed of a function system and 2 relationship systems. The function system contains the physiological function and pathological changes of all viscera. The 2 relationship systems include the correlations between the 5 zang organs and the body, organs, and orifices (竅, such as tongue, ears, eyes, nose, mouth, external genitals, 
Liao et al.: The Brief Theory of Viscus and Its Manifestations in Traditional Chinese Medicine

and anus), the 5 zang organs and the 5 constituents (namely vessels, tendons, muscles, skins, and bones), and the correlation between the zang organs and the 6 fu organs [4].

The 5 zang organs and the 6 fu organs are all internal organs, but they differ in functions and characteristics. The main function of the 5 zang organs is “to store jing-qi (essence, 精氣)" which is the refined food nutrient responsible for maintenance of life activities. The main function of the $6 \mathrm{fu}$ organs is "to transport and transform food." That means to receive and digest food, absorbing the nutrients and discharging the waste. That is why TCM has divided the internal organs into 2 distinct categories: the zang organs and the fu organs.

Zang organs and fu organs control all life activities. The substantial bases responsible for the physiological functions of the 5 zang organs and the 6 fu organs are founded on 2 aspects: one is morphological structure in anatomy and the other is refined nutrient like qi, blood, yin, and yang. A different morphological structure bears a certain relationship to the physiological functions of certain internal organs. The theory of TCM pays more attention to the synthetic manifestations of the holistic functions of the body and is more sophisticated in distinguishing the intrinsic nature of life from the external manifestation. The zangxiang theory claims that qi, blood, yin, and yang are the essential substances for constituting and maintaining the physiological functions of the zang-fu organs. All pathological changes result from deficiency or disturbance of these substances. Substantially, these refined substances are fine and minute. Though invisible to the eyes, they can still be understood and distinguished according to their physiological functions and pathologic changes by the TCM doctors and researchers. Generally, qi and yang both have the functions of warming, propelling, and fixating. However, the function of qi is to propel and fixate, while the function of yang is to warm the whole body. Both TCM blood and yin have the functions of nourishing and moistening like the blood with the contents (such as oxygen, nutrition, etc.) of Western medicine. However, in TCM, the function of blood is to nourish while yin has the task to moisten. Qi, blood, yin, and yang differ in qihua (qi-transformation). That is why they bear different physiological functions.

Overall, the zangxiang theory focuses on the study of physiological functions and pathological changes of the zang-fu organs, somatic tissues, and organs as well as the mutual relationships among them. Therefore, it is the core of the theoretical system of TCM. Though terminologically identical to some of the organs in modern medicine, in the zangxiang theory, the zang-fu organs are quite different in its physiological functions. The zangxiang theory was established mainly on the basis of life experience and clinical practice. The conceptions of zang-fu organs in TCM are quite beyond the range of anatomical morphology. It is generally believed that the functions of the zang-fu organs in TCM are extensive, not only including part of the functions of the organs with the same names in modern medicine, but also covering certain functions of other related organs. Obviously, the zang-fu organs in TCM are not only the conceptions of anatomy but synthetic functional units.

\section{The Five Zang Organs, Its Constituents, and Its Orifices}

The 5 zang organs include heart, lung, liver, spleen, and kidney. The theory does not only describe the physiological functions of the 5 zang organs but also shows their relationships between the organs and the body, the organs and the orifices, and also describes the relationship between the 5 zang organs and the 5 constituents, the 5 zang organs, and the focal external manifestations.

The 5 constituents include the vessels, the skin, the tendons, the muscles, and the bones that are dominated by the 5 zang organs, respectively. The orifices, simplification for "the 5 sensory organs and the 9 orifices," include the tongue, the nose, the eyes, the ears, the external genitals, and the anus. The heart, the lung, the liver, and the spleen govern 1 orifice, respec- 
Liao et al.: The Brief Theory of Viscus and Its Manifestations in Traditional Chinese Medicine

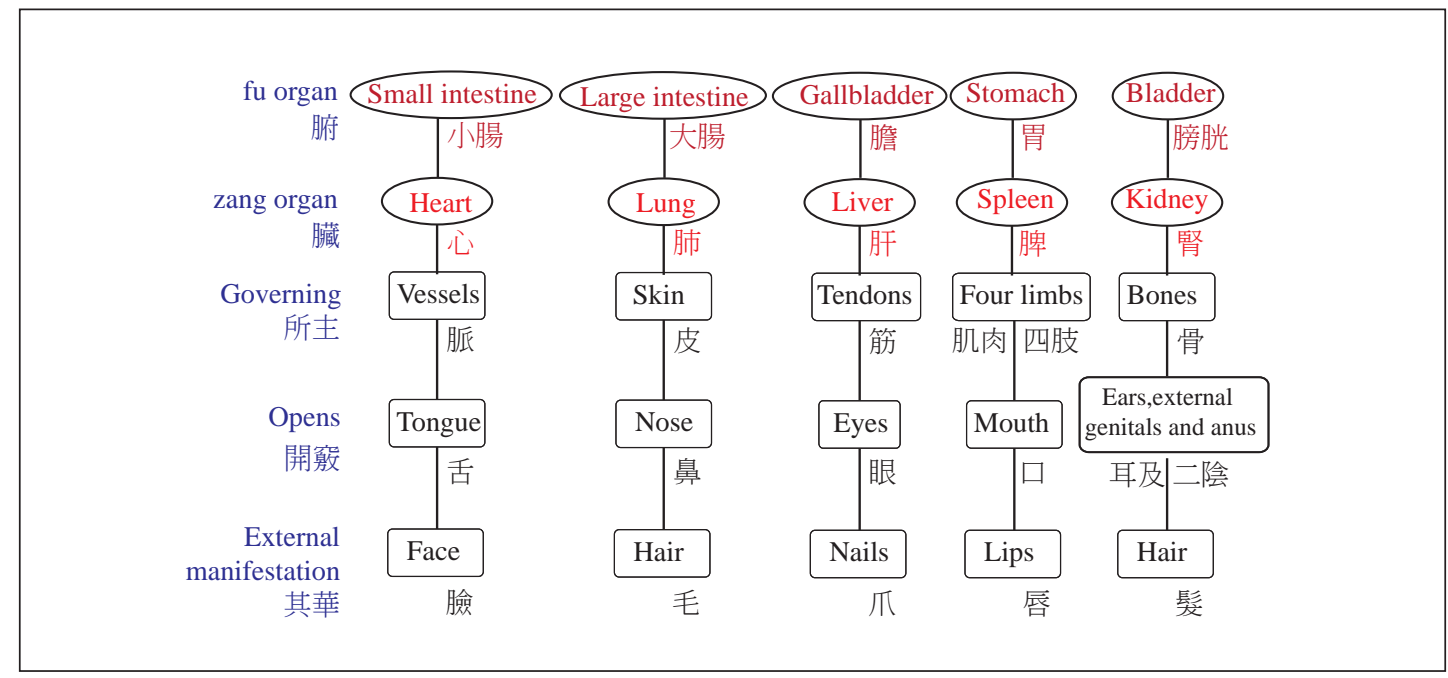

Fig. 1. The relationships of the zang organs, fu organs, its sensory organs, and the orifices.

tively. However, the kidney opens into the ears, the external genitals, and the anus. The focal external manifestations of the 5 zang organs refer to 5 special areas (i.e., the face, the body hair, the tendons, the lips, and the hair) on the surface of the body on which the essence of the 5 zang organs is shown.

The relationship of the zang organs, fu organs, its sensory organs, and the orifices is shown in Figure 1.

The Heart

The physiological functions of the heart are mainly to govern the blood and control the mind, and sometimes to control emotions and feelings. The heart governs the vessels, opens into the tongue, with external manifestation on the face.

\section{The Pericardium}

It is an appendix organ that surrounds the heart like a tissue to protect it. The zangxiang theory states that the heart is the monarch organ and cannot be directly attacked by pathogenic factors. If pathogenic factors directly invade the heart, it will disturb the heart-spirit and threaten life. For example, the symptoms of high fever, coma, and delirium in exogenous febrile diseases are described as "invasion of pathogenic factors into the pericardium" in TCM.

\section{The Lung}

It is located in the chest and is the highest among the internal organs. The function of the lung mainly depends on the propelling and fixating functions of lung-qi and on the moistening function of lung-yin. The lung may dominate the qi and manage the regulation of water passage and govern the skin. The main task of the skin is to protect the body, excrete sweat, and adjust the body temperature. The lung governs the hair, i.e. the lung has its external manifestation on the hair. The lung opens into the nose for smelling.

The Liver

It is located below the diaphragm and on the right side of the ribs as in Western medicine. The liver stores yin-blood and has a function as yang because yang-qi in the liver is very active 
and resolute, tending to disperse. The liver possesses 2 physiological functions: the first is to govern dredging and regulate materials (including blood, metabolism, yin, yang, and qi, etc.) of the body (it is called shu-xie function in TCM), and the second is to store blood. It can also assist the spleen and the stomach to digest food by modifying the spleen-qi and the stomachqi with its dredging and regulating functions, and the liver can control mental activity such as joy, anger, anxiety, and contemplation, etc. Finally, the liver can regulate menstruation in women. The liver governs the tendons and opens into the eyes. The external manifestation of the liver is on the nails.

\section{The Spleen}

It is located in the abdomen and governs digestion and absorption. As the source of qi, blood, and body fluid, the spleen plays a vital role in maintaining life activities. The spleen governs transportation and transformation, it means the spleen can digest food, absorb nutrients of food and water, and then transport them to the heart and the lung. It can command blood as to control the blood circulation inside the vessels and prevent it from flowing out of the vessels and govern the muscles and the 4 limbs. The spleen opens into the mouth, and its external manifestation is on the lips.

\section{The Kidney}

It is located in the waist, and the handbook Huangdi Neijing says that "the waist is the house of the kidney." The kidney stores essence and the essence transforms qi and produces blood. It is the source of genuine yin and genuine yang, and it is related with the essence, qi, blood, yin, and yang. The physiological functions of the kidney are composed of 6 parts: to govern growth and development, to govern reproduction, to govern water (modify and maintain all fluid of the body), to govern reception of qi, to produce marrow to enrich the brain and transform blood, and to nourish and warm the internal organs. The bones (including the growth of teeth) are governed by the kidney. It opens into the ears, the external genitals, and the anus, and their external manifestation is on the hair.

\section{The Interactions of the Zang Organs and the Fu Organs in the Five Elements Theory}

The 5 elements theory (五行), originating in ancient China, is the theory of yin and yang. It includes 5 basic elements of the earth, which also form the body of human beings. In ancient times, this theory was used to explain and analyze things and their changes in the natural world. The theory of the 5 elements was applied to demonstrated the interactions of zang organs and fu organs in the theoretical system of TCM (Fig. 2). It includes 2 main interactions: interpromotion (相生) and interrestraint (相剋). Interpromotion means that one thing bears the effect of promoting or generating another in the 5 elements theory. For example, the zangorgan liver (which has the property of wood in the 5 elements theory) can promote the zangorgan heart (which has the property of fire in the 5 elements theory), and at present we say "wood promoting fire." Similarly, the zang-organ kidney (which has the property of water in the 5 elements theory) can promote the zang-organ liver (which has the property of wood in the 5 elements theory), and at present we say "water promoting wood." Interrestraint means that one thing controls and restrains another thing in the 5 elements theory. The property of liver as wood may restrain the spleen whose property is earth, spleen (earth) restraining kidney (water), kidney (water) restraining heart (fire), heart (fire) restraining lung (metal), and the lung (metal) restraining liver (wood). 
Liao et al.: The Brief Theory of Viscus and Its Manifestations in Traditional Chines Medicine

Fig. 2. The interactions of the zang organs and the fu organs in the 5 elements theory.

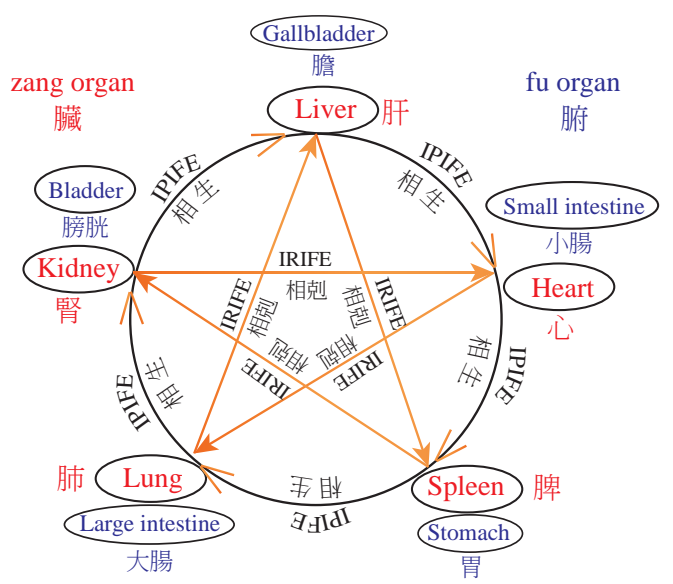

IPIFE (相生): inter-promotion in five elements

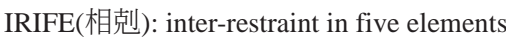

Interpromotion and interrestraint among the 5 elements are in fact inseparable. Without promotion, nothing can grow and develop; without restraint, there will be no way to prevent harm caused by excessive development of things. Only when restraint exists in promotion and promotion in restraint can the normal development and harmonious balance of things be maintained.

\section{Conclusion}

Zangxiang and other TCM theories, such as yin and yang, the 5 elements theory, and the liu jing bian zheng theory, etc., are very important constituent parts for understanding the physiological functions and pathological changes of the TCM system. In modern society, the basic theory of TCM may direct TCM doctors or researchers to combine it with the modern medicine system in order to develop and improve it and adapt the needs of the integrative medicine area. A better understanding of zangxiang can illustrate it more comprehensively, accurately, and systematically and can help in disseminating TCM in countries and regions where English is the official language.

\section{Acknowledgment}

This study was sponsored and supported by the National Natural Science Foundation of China (81373619).

\section{Disclosure Statement}

The authors declare that there are no conflicts of interest regarding the publication of this paper. 

www.karger.com/imi

Liao et al.: The Brief Theory of Viscus and Its Manifestations in Traditional Chinese Medicine

\section{References}

1 Pan W, Zhou H: Inclusion of integrative medicine in clinical practice. Integr Med Int 2014;1:1-4.

-2 Zhou WT, Jia SY, Zhang YQ, Tian YY, et al: Pathological changes in internal organs after blocking low hydraulic resistance channels along the stomach meridian in pigs. Evid Based Complement Alternat Med 2013;2013: 935687.

-3 Yang X, Jia C: Understanding association of spleen system with earth on traditional Chinese medicine theory. J Tradit Chin Med 2013;33:134-136.

4 Zhang L, Zhang QM, Wang YG, Yu DL, et al: The TCM pattern of the six-zang and six-fu organs can be simplified into the pattern of five-zang and one-fu organs. J Tradit Chin Med 2011;31:147-151. 